\title{
THE IMPACT OF ENVIRONMENTAL TOBACCO SMOKE EXPOSURE ON CARDIORESPIRATORY FITNESS IN CHILDREN: A PILOT STUDY
}

\author{
MELISSA PARNELL, IVAN GEE, LAWRENCE FOWEATHER, GREG WHYTE, \\ ZOE KNOWLES \& JOHN DICKINSON \\ Public Health Institute \& Physical Activity Exchange, Liverpool John Moores University, UK
}

\begin{abstract}
Environmental tobacco smoke (ETS) in indoor air is a substantial risk factor for many health issues. Children are particularly susceptible to ETS with increased risk of asthma attacks, respiratory infections and sudden infant death syndrome. The health effects of ETS are well researched in adults, but few studies examine the impact on children's cardiorespiratory fitness (CRF). CRF has been shown to be a useful biomarker for monitoring health effects which would normally be too subtle to identify at rest. In adults, ETS has been shown to reduce CRF, and children may be at greater risk due to high respiration rates and developing organs. This preliminary research tests the hypothesis that ETS has a detrimental impact on CRF in children. Twenty-five children (9-11 years) from one Merseyside primary school were recruited. ETS exposure was determined by parental surveys and coupled with children's exhaled carbon monoxide concentration. CRF was determined using a $\mathrm{VO}_{2}$ peak test, with lung function assessed using standard spirometry, and fractional exhaled nitric oxide (FeNO) provided an indication of lung inflammation. Initial results show that children exposed to ETS had statically lower CRF scores $(p=0.048)$ and were more likely to be classified as 'unfit' compared to children not exposed. A negative correlation was found between the number of cigarettes smoked at home and children's CRF ( $r=-0.526, p=0.008)$, suggesting a possible dose-response relationship. Spirometry and $\mathrm{FeNO}$ values were not statistically different between groups. Results indicate that ETS exposure is likely to be detrimental to children's CRF. They highlight the need for further work, on a larger dataset that will allow more robust analysis with greater statistical power. To the author's knowledge, this study is the first of its kind to use laboratory-based fitness measurements to explore associations between ETS and CRF in children.
\end{abstract}

Keywords: cardiorespiratory fitness, children, environmental tobacco smoke, FeNO, indoor air pollution, spirometry, tobacco

\section{INTRODUCTION}

Approximately $83 \%$ of tobacco smoke is in an invisible and gaseous form [1] which inadvertently exposes non-smokers to the effects of environmental tobacco smoke (ETS), often referred to as second-hand smoke (SHS), a common and significant indoor air pollutant. The effects of second-hand smoking are substantial and rapid, with effects predicted to be almost as large as chronic active smoking [2]. In adults, ETS can cause coronary heart disease, stroke and lung cancer, and exposed children can suffer from numerous health problems including severe asthma attacks, respiratory infections, ear infections and sudden infant death syndrome [3]. Like all carcinogens, there is no risk-free level of SHS exposure [4]. Worldwide, approximately $34 \%$ of non-smoking adults and $40 \%$ of children are exposed to SHS [5]. Öberg et al. calculated that in 2004, 603,000 deaths (approximately 1\% of worldwide mortality) were attributed to ETS and 10.9 million disability-adjusted life years. Two main constituents of cigarette smoke, nicotine and carbon monoxide (amongst others), exhibit toxic effects on cardiovascular function both at rest and during exercise in adults [6]. The risk from ETS exposure is time dependant, and therefore, limiting exposure early on is the most effective strategy for a reduction in tobacco smoke morbidity and mortality [7]. Children are particularly susceptible to the effects of ETS due to their high respiratory rates and immature 
organs [8], and therefore, extra effort should be taken to safeguard children from second-hand tobacco smoke.

Physical fitness is an integrated measure of most body functions involved in the performance of daily physical activity (PA). Therefore, the functionality of all these systems is being checked when physical fitness is tested [9]. Cardiorespiratory fitness (CRF) is a healthrelated component of physical fitness defined as the ability of the circulatory, respiratory and muscular systems to supply oxygen during sustained PA [10]. The maximal oxygen consumption $\left(\mathrm{VO}_{2} \max \right)$ attained during a graded maximal exercise to voluntary exhaustion has long been considered by the World Health Organisation as the single best indicator of CRF [11]. There is robust evidence that poor CRF is an independent risk factor for obesity-, diabetes- and heart disease-related mortality and that a moderate to high level of CRF reduces the risk of all-cause cardiovascular disease mortality [12], [13].

Subtle changes in the function of the cardiorespiratory system can be recognised through exercise-based measures. Therefore, exercise-related health markers, such as $\mathrm{VO}_{2}$ peak, may provide novel methods for measuring health in relation to ETS exposure in children. To date, no previous research has sought to examine the impacts of ETS exposure on children's laboratory-measured $\mathrm{VO}_{2}$ peak, and therefore, the present study will provide new insight to current body of knowledge. Using a small initial sample population, this exploratory study aims to examine the hypothesis that exposure to the indoor air pollutant, ETS, is detrimental to children's CRF.

\section{METHOD}

\subsection{Recruitment}

Prior to recruitment, ethical approval was obtained from Liverpool John Moores University Ethics Committee. Children from one Liverpool (United Kingdom) primary school, ages 9-11 years $(n=25)$, agreed to take part in the study. Inclusion criteria were children aged 9-11 years old and attend a Merseyside primary school. Exclusion criteria were any medical conditions that limit a child's ability to walk or run on a treadmill, including heart conditions where vigorous exercise would put the child at risk.

\subsection{Quantitative surveys}

Questionnaires to determine self-reported household smoking status, adapted from the Global Adult Tobacco Survey [14], were completed by a consenting parent or guardian. Questions determined the number of tobacco smokers in the home, as well as which rooms smoking occurred, and how many cigarettes were smoked each day. Participant (adult and child) demographic information was also obtained via the parental questionnaire, with postcodes used to determine English indices of multiple deprivation score (EIMD) [15]. Children completed a Physical Activity Questionnaire for Children (PAQ-C) to determine the self-reported levels of PA.

\subsection{Anthropometrics}

Body mass to the nearest $0.1 \mathrm{~kg}$ (Seca, Birmingham, UK), stature and sitting stature to the nearest $0.1 \mathrm{~cm}$ (Seca, Birmingham, UK) were assessed using standard techniques [16]. Body 
mass index (BMI) was calculated from height and weight $\left(\mathrm{kg} / \mathrm{m}^{2}\right)$, with age- and sex-specific BMI cut-points used to classify child weight status [17].

\subsection{Exhaled gases}

Exhaled carbon monoxide (eCO) was measured using a breath Smokerlyzer PiCO device (Bedfont, UK) to determine recent ETS exposure. Pilot work indicated that eCO quickly declined several hours post-ETS exposure. Therefore, eCO measurements were taken on the morning (08:30) of laboratory visits to better reflect ETS exposure from the home. Fractional exhaled nitric oxide (FeNO), an indication of airway inflammation, was measured at rest (twice) using a NIOX ${ }^{\circledR}$ VERO device (Circassia, UK) in the laboratory, prior to spirometry.

\subsection{Spirometry}

Spirometry measures, such as forced vital capacity $(\mathrm{FVC})$, forced expiratory volume $\left(\mathrm{FEV}_{1}\right)$, peak expiratory flow (PEF) and forced expiratory ratio (FER), were taken at rest using a digital micro-spirometer (Micro-plus spirometer, CareFusion, UK). Spirometry measures were repeated at least three times with the highest value recorded.

\subsection{Cardiorespiratory fitness}

CRF, peak oxygen uptake ( $\mathrm{VO}_{2}$ peak), was assessed using an individually calibrated, continuous incremental treadmill test to volitional exhaustion using breath-by-breath analysis (Jaeger Oxycon Pro, Viasys Health Care, UK). This method is based on an established protocol described previously by Boddy et al [18]. $\mathrm{VO}_{2}$ peak, as opposed to $\mathrm{VO}_{2}$ max (plateau of oxygen uptake), was used as children often fail to reach plateau [19]. However, experiments have shown that 'true' $\mathrm{VO}_{2}$ max values can be achieved in children without the need for plateau if test endpoints are met [20].

\subsection{Analysis}

Data were analysed using a combination of Microsoft Office Excel 2016 and IBM Statistical Package for the Social Sciences (SPSS) v24. Incomplete datasets and participants which did not reach peak $\mathrm{VO}_{2}$ [18] were excluded from the analysis of CRF.

\section{RESULTS}

\subsection{Participant characteristics}

Of the 25 children who returned written parental informed consent and participant assent, only one participant did not complete the $\mathrm{VO}_{2}$ peak fitness test. Participant response rate for the one participating school was $40.9 \%$. It should be noted that the recruited number of participants from smoking households was very low ( $n=2$ females, $n=5$ males) and so thorough analysis of these individual groups is limited. Both the school and participant's homes are urban within the Liverpool City air quality management area and are included in the $20 \%$ most deprived neighbourhoods in England. White British children accounted for $88 \%$ of the sample, with $8 \%$ Polish and $4 \%$ Portuguese ethnicity. 
Males were found to have significantly higher $\mathrm{VO}_{2}$ peak scores than females $(p=0.042)$. Due to the finding, the dataset was split by sex for certain aspects of the analysis. To allow for male and female datasets to be analysed together, children were classified as aerobically fit or unfit according to the established thresholds for male and female children, 46.6 and $41.9 \mathrm{ml} /$ $\mathrm{min} / \mathrm{kg}$ for males and females, respectively [21].

\subsection{Breath eCO as a measure of environmental tobacco smoke exposure}

A moderate, statistically significant Pearson correlation $(r=0.504, p=0.010)$ was found between eCO concentration and parental self-reported number of cigarettes smoked per day. Children with self-reported ETS exposure had a higher mean eCO of $1.57 \mathrm{ppm}$ compared to those not exposed, $1.00 \mathrm{ppm}$, although this was not statistically significant $(p=0.219)$.

\subsection{Spirometry and FeNO}

No statistically significant differences were found for spirometry measures between exposure groups (Table 1). Mean fractional nitric oxide concentration in exhaled breath (FeNO) in the smoking group was almost double that of the non-smoking group (22 ppb and $40.1 \mathrm{ppb}$, respectively), but this was not found to be statistically significant $(p=0.178)$. The range for FeNO measurements was very high (140) with a minimum value of $5 \mathrm{ppb}$ and a maximum of $145 \mathrm{ppb}$.

\section{4 $\mathrm{VO}_{2}$ peak between exposure groups}

Fitness ( $\mathrm{VO}_{2}$ peak) was found to be significantly lower in the group exposed to ETS with a mean $\mathrm{VO}_{2}$ peak of 50.64 and $44.64(\mathrm{ml} / \mathrm{min} / \mathrm{kg})$, respectively, for children exposed and not exposed to ETS $(p=0.048)$. However, when the same analysis was performed separately for males and females, only the difference between the male exposure groups was significant (Table 2).

Table 1: Mean spirometry values expressed as percentage of normative values for sex, height and ethnicity. FVC - Forced vital capacity, FEV1 - Forced expiratory volume in 1 second, PEF - Peak expiratory flow, FER - Fractional exchange ratio, FeNO Fraction of exhaled nitric oxide.

\begin{tabular}{lccc}
\hline $\begin{array}{l}\text { Measure } \\
(\text { mean })\end{array}$ & $\begin{array}{c}\text { Non-smoking household } \\
(n=18)\end{array}$ & $\begin{array}{c}\text { Smoking household } \\
(n=7)\end{array}$ & $T$-test $p$ values \\
\hline$\%$ FVC & $97.7(18.1)$ & $92.7(24.6)$ & 0.580 \\
$\%$ FEV $_{1}$ & $86.0(20.8)$ & $88.9(19.2)$ & 0.754 \\
$\%$ PEF & $88.2(31.3)$ & $82.6(22.1)$ & 0.743 \\
$\%$ FER & $92.2(10.3)$ & $98.4(9.62)$ & 0.178 \\
FeNO $(\mathrm{ppb})$ & $22.0(19.6)$ & $40.1(47.0)$ & 0.178 \\
\hline
\end{tabular}


Table 2: Mean $\mathrm{VO}_{2}$ peak scores by sex for children exposed and not exposed to ETS in the home.

\begin{tabular}{lccc}
\hline & \multicolumn{2}{c}{ Mean $\mathrm{VO}_{2}$ peak $\pm \mathrm{SD}$} & Statistical significance \\
\cline { 2 - 3 } & Exposed $(n=7)$ & Not exposed $(n=17)$ & \\
\hline Male & $46.28(6.95)$ & $55.47(3.96)$ & $p=0.015$ \\
Female & $40.55(8.27)$ & $47.25(4.69)$ & $p=0.125$ \\
\hline
\end{tabular}

A moderate $(r=-0.470)$ yet significant $(p=0.021)$ negative correlation (Pearson) was found between eCO and $\mathrm{VO}_{2}$ peak when both sexes were analysed together (Fig. 1). The same significance was not found when the data were split by sex. A negative correlation was also found between parental self-reported number of cigarettes smoked per day and $\mathrm{VO}_{2}$ peak, $r=-0.526, p=0.008$. Both sexes showed a reduction in fitness as the number of cigarettes smoked at home per day increased, with Pearson $r=-0.635, p=0.026$ for males, and $r=-0.630, p=0.028$ for females. The correlation (Pearson) no longer held statistical significance when the extreme value at 65 'cigarettes smoked per day' was removed, $r=-0.314$, $p=0.145$. However, when split by sex, the trend was still apparent for males, $r=-0.635$, $p=0.026$.

More children were classed as fit from non-smoking households (94\%) compared to smoking households (29\%) where the majority were classed as aerobically unfit (Chi-square test, $p=0.001$ ), as shown in Table 3. Fitness thresholds were 46.6 and $41.9 \mathrm{ml} / \mathrm{min} / \mathrm{kg}$ for males and females, respectively.

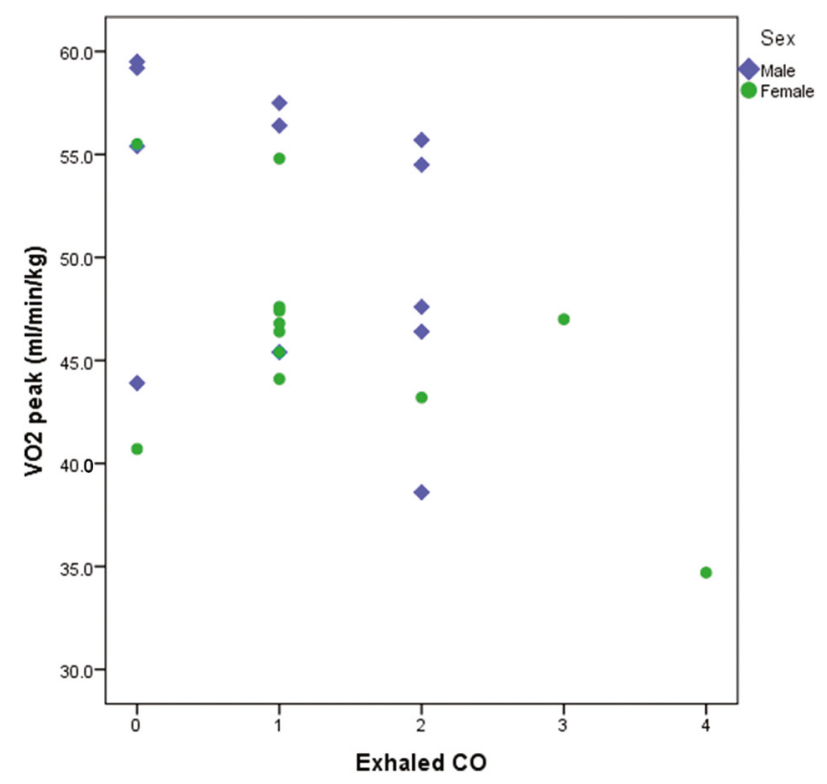

Figure 1: $\mathrm{VO}_{2}$ peak $(\mathrm{ml} / \mathrm{min} / \mathrm{kg})$ and eCO $(\mathrm{ppm})$ for males and females. 
Table 3: Percentage of children classed as fit from smoking $(n=7)$ and non-smoking households $(n=17)$.

\begin{tabular}{lcc}
\hline & Fit $(\%)$ & Unfit $(\%)$ \\
\hline Smoking household $(n=7)$ & 29 & 71 \\
Non-smoking household $(n=17)$ & 94 & 6 \\
\hline
\end{tabular}

Table 4: Mean BMI scores for males and females from smoking and non-smoking households.

\begin{tabular}{lccc}
\hline & \multicolumn{2}{c}{ Mean BMI \pm SD } & \\
\cline { 2 - 3 } & Exposed & Not exposed & Statistical significance from $t$-test \\
\hline Male & $20.05(3.39)$ & $16.78(1.25)$ & \\
Female & $20.17(4.51)$ & $19.00(3.60)$ & $p=0.044$ \\
\hline
\end{tabular}

\subsection{Body mass index}

A significant negative correlation (Pearson) was found between $\mathrm{BMI}$ and $\mathrm{VO}_{2}$ peak for both males $(r=0.876, p<0.001)$ and females $(r=-0.800, p=0.002)$. The mean BMI was higher in the ETS-exposed group, but this was only statistically significant for males (Table 4).

\subsection{Deprivation}

Deprivation score, EIMD, was negatively correlated with $\mathrm{VO}_{2}$ peak fitness score for males only $(r=-0.683, p=0.020)$. EIMD scores were found to be significantly higher for smoking families, 57.4, than non-smoking families, $41.2(p=0.023)$.

\subsection{Physical activity}

The mean self-reported PA scores were 3.59 (SD 0.65) for females and 3.46 (SD 0.70) for males ( 1 indicating low PA and 5 high PA). PA scores showed no correlation with $\mathrm{VO}_{2}$ peak scores $(r=-0.001, p=0.997$ for males, and $r=0.087, p=0.780$ for females).

\section{DISCUSSION}

This initial study examined the association between exposure to ETS in the home and children's CRF from a small sample of children from one Liverpool primary school. A significant difference in CRF was found between the children from smoking and non-smoking homes with $71 \%$ of the children from smoking homes being classed as aerobically unfit compared to only $6 \%$ from non-smoking households. The mean CRF, as measured by $\mathrm{VO}_{2}$ peak, was $16 \%$ lower in male children from smoking households and $6.7 \%$ lower in females from smoking households compared to non-smoking households. However, $51 \%$ of the children from smoking households were classed as being either overweight or obese compared to $22 \%$ of the children from non-smoking homes, which could be an important confounder. 
Self-reported PA score was not found to be correlated with $\mathrm{VO}_{2}$ peak, suggesting that fitness is influenced by other factors. However, self-reporting surveys are notoriously unreliable and further confounded by the fact the participants are children and may exaggerate their PA levels due to enthusiasm or desirability bias.

Exhaled CO was positively correlated with the number of cigarettes parents reported smoking at home each day. Both eCO and cigarettes smoked per day were negatively correlated with CRF in males and females. The results of the present study suggest that tobacco smoking, and the resulting CO inhalation, may be having a detrimental effect on children's CRF, as found previously in adults [22]. However, due to the nature of this study and the small sample size, causation cannot be assumed. ETS is not the only source of CO. The road transport microenvironment is a significant contributor to an individual's $\mathrm{CO}$ exposure, with travel by vehicle causing the highest level of CO exposure compared to walking and cycling [23]. With this in mind, particle monitors (Dylos 1700) will be set up in homes and schools of smoking and nonsmoking participating families to determine $\mathrm{PM}_{2.5}$ concentrations in the home. This information will be used to compare the air quality in smoking and non-smoking homes and to highlight any other significant PM exposures (e.g. traffic congestion near the home or school).

In the present study, FeNO values were highly variable with a range of 140, the lowest value at $5 \mathrm{ppb}$, and the highest at $145 \mathrm{ppb}$. Eosinophilic inflammation is likely at $35 \mathrm{ppb}$ or higher [24]. Repeat measures on individuals only varied by 1-2 ppb; therefore, the range is due to individual differences. No statistically significant associations were found between the exposure groups and FeNO, although the tobacco smoking group did have double the percentage of children classified as having a high concentration ( $>35 \mathrm{ppb}$ ) of FeNO compared to the nonsmoking group. There are a number of conditions that elevate levels of FeNO, including asthma and allergic rhinitis [25]. A larger dataset is needed to better determine whether elevated concentrations of FeNO are associated with ETS; this will be explored in future work.

Spirometry measures were expressed as a percentage of the normative values for age, height, sex and ethnicity. Mean \%FVC and mean \%PEF were, respectively, 5.0\% and 5.6\% lower in the smoking group compared to the non-smoking group, but this was not statistically significant. Parental smoking has been shown to reduce lung function in children, as measured through spirometry. For example, a small but significant decrease in $\mathrm{FEV}_{1}$ due to parental smoking and in utero exposure has been associated with reduced PEF and FVC [26], [27]. A major limitation with the use of spirometry with children is compliance; children often find it difficult to perform the test correctly and therefore recorded values may not reflect true ability. As described earlier, some subtle changes in system functions can only be picked up through exercise-related measures. All spirometry measures in the present study were completed with the participant at rest, and further work will aim to include mid-flow spirometry measures during exercise which may be a more sensitive measure of lung function and health.

The present study shows that unfit males on average have higher deprivation (EIMD) scores. Children from more deprived households may have less opportunity for organised PA, which may explain the reduced $\mathrm{VO}_{2}$ peak, but it may be the case that smoking habits, which are also associated with deprivation, are partly responsible for the difference. As EIMD scores are calculated using postcodes, and fitness was correlated to EIMD, local air quality may be also influencing children's fitness. Liverpool City is an Air Quality Monitoring Area with regular exceedances of national standards for nitrogen dioxide. However, the nearest automatic urban monitoring network station is Speke, Liverpool, approximately $10 \mathrm{~km}$ from the participating school. Whilst these data could be utilised, they would not very well reflect the children's exposure from their microenvironment, especially not in the home. As stated above, further 
work will use $\mathrm{PM}_{2.5}$ monitors to assess air quality both in the home and at the school, which will shed light on the contribution from other sources of air pollution, as well as indoor ETS.

The number of females from smoking households was extremely low in this sample $(n=2)$, and therefore, statistical analysis of this group is limited. Participants from smoking households accounted for $28 \%$ of the sample, with the majority reporting to smoke 20 or less cigarettes per day, and one participating family smoking at 65 per day. It would be useful to recruit children from households who smoke more than 20 cigarettes per day, which might elude to a linear or otherwise trend between child CRF and household cigarettes smoked per day. Whilst the ratio of males to females was almost 50\%, white British children accounted for $88 \%$ of the participants in the current study, and further work will include participants from a variety of ethnic backgrounds.

ETS poses a significant threat to an individual's health, with children at a greater risk due their developing organs. CRF is a good indicator of general health as it encompasses all of the body's major systems and organs [9]. An overweight and unfit child is likely to become an overweight and unfit adult [28], which is of great concern for the future of public health. The current study has provided an insight into ETS exposure and the potential detrimental impact on children's CRF. This study has demonstrated a significant difference between the two exposure groups with a small sample size. It will be of great interest and importance to see if these differences stand true with a larger and more diverse sample.

\section{ACKNOWLEDGEMENTS}

This study was funded by Liverpool John Moores University FREE PhD Studentship Fund.

\section{REFERENCES}

[1] Gee, I.L., Semple, S., Watson, A. \& Crossfield, A., Nearly $85 \%$ of tobacco smoke is invisible, a confirmation of previous claims. Tobacco Control, 22, p. 429, 2013.

[2] Barnoya, J. \& Glantz, S.A., Cardiovascular effects of second-hand smoke: nearly as large as smoking. Circulation, 111, pp. 2684-2698, 2005.

[3] Naeem, Z., Second-hand smoke - ignored implications. International Journal of Health Sciences, 9, pp. V-VI, 2015.

[4] Centers for Disease Control and Prevention (US) \& U.S. Department of Health and Human Services. The Health Consequences of Involuntary Exposure to Tobacco Smoke: A Report of the Surgeon General. Publications and Reports of the Surgeon General, 2006.

[5] Öberg, M., Jaakkola, M.S., Woodward, A., Peruga, A. \& Prüss-Ustün, A., Worldwide burden of disease from exposure to second-hand smoke: a retrospective analysis of data from 192 countries. Lancet, 377, pp. 139-146, 2011.

[6] Papathanasiou, G., Mamali, A., Papafloratos, S. \& Zerva, E., Effects of smoking on cardiovascular function: the role of nicotine and carbon monoxide. Health Science Journal, 8, pp. 272-288, 2014.

[7] Tantucci, C. \& Modina, D., Lung function decline in COPD. International Journal of Chronic Obstructive Pulmonary Disease, 95, 2012. DOI: 10.2147/COPD.S27480.

[8] Lonngman, J. \& Passey, M., Children, smoking households and exposure to secondhand smoke in the home in rural Australia: analysis of a national cross-sectional survey. BMJ Open, 3, p. e003128, 2013.

[9] Ortega, F.B., Ruiz, J.R., Castillo, M.J. \& Sjöström, M., Physical fitness in childhood and adolescence: a powerful marker of health. International Journal of Obesity, 32, pp. 1-11, 2008. 
[10] Lee, D., Artero, E.G., Xuemei Sui \& Blair, S.N., Review: Mortality trends in the general population: the importance of cardiorespiratory fitness. Journal of Psychopharmacology, 24, pp. 27-35, 2010.

[11] Shephard, R. J. et al., The maximum oxygen intake. An international reference standard of cardiorespiratory fitness. Bulletin of the World Health Organization, 38, pp. 757764, 1968.

[12] Kokkinos, P. et al., Exercise capacity and mortality in black and white men. Circulation, 117, pp. 614-622, 2008.

[13] Dencker, M. et al., Aerobic fitness related to cardiovascular risk factors in young children. European Journal of Pediatrics, 2012. DOI: 10.1007/s00431-011-1617-0.

[14] Global Adult Tobacco Survey Collaborative Group. Participatory Drawing as a Visual Research Method with Children and Youth, 2011.

[15] Office for National Statistics. Do Smoking Rates Vary Between More and Less Advantaged Areas. The National Archives, 2014 (accessed 12 February 2018).

[16] Lohman, T.G., Roche, A.F. \& Martorell, R., Anthropometric standardization reference manual. Journal of Human Kinetics, 177, 1988. DOI: 10.1002/ajhb.1310040323.

[17] World Health Organisation. BMI for age 5-19 years. Growth reference 5-19 years, available at http://www.who.int/growthref/who2007_bmi_for_age/en/, 2007 (accessed 26 February 2019).

[18] Boddy, L.M. et al., Physical activity, cardiorespiratory fitness, and clustered cardiometabolic risk in 10- to 12-year-old school children: The REACH Y6 study. American Journal of Human Biology, 26, pp. 446-451, 2014.

[19] Armstrong, N. \& van Mechelen, W., Oxford Textbook of Children's Sport and Exercise Medicine. 3rd ed. Oxford, United Kingdom: Oxford University Press, 2017.

[20] Armstrong, N. \& Winsley, R., Is Peak VO, a maximal index of children's aerobic fitness? International Journal of Sports Medicine, 17, pp. 356-359, 1996.

[21] Boddy, L.M. et al., ROC generated thresholds for field-assessed aerobic fitness related to body size and cardiometabolic risk in schoolchildren. PLoS One 7, p. e45755, 2012.

[22] De Borba, A. T. et al., The influence of active and passive smoking on the cardiorespiratory fitness of adults. Multidisciplinary Respiratory Medicine, 9, p. 34, 2014.

[23] Kaur, S., Nieuwenhuijsen, M.J. \& Colvile, R.N., Fine particulate matter and carbon monoxide exposure concentrations in urban street transport microenvironments. Atmospheric Environment, 41, pp. 4781-4810, 2007.

[24] Dweik, R. A. et al., An official ATS clinical practice guideline: interpretation of exhaled nitric oxide levels (FENO) for clinical applications. American Journal of Respiratory and Critical Care Medicine, 2011. DOI: 10.1164/rccm.9120-11ST.

[25] Chan, E.Y., Ng, D. \& Chan, C.H. Measuring FENO in asthma: coexisting allergic rhinitis and severity of atopy as confounding factors. American Journal of Respiratory and Critical Care Medicine, 180, p. 281, 2009.

[26] Cook, D.G., Strachan, D.P. \& Carey, I.M. Health effects of passive smoking. 9. Parental smoking and spirometric indices in children. Thorax, 53, 884-93, 1998.

[27] Gilliland, F.D. et al., Maternal smoking during pregnancy, environmental tobacco smoke exposure and childhood lung function. Thorax, 55, pp. 271-276, 2000.

[28] Deshmukh-Taskar, P. et al., Tracking of overweight status from childhood to young adulthood: the Bogalusa Heart Study. European Journal of Clinical Nutrition, 60, pp. 48-57, 2006. 